DOI: $10.3901 / J M E .2021 .08 .090$

\title{
结构自适应频谱塑形主动控制研究*
}

\author{
刘金金金 1,2 陈雪峰 ${ }^{1,2}$ 张兴武 ${ }^{1,2}$ 乔百杰 ${ }^{1,2}$ 严如强 1,2
}

(1. 西安交通大学机械工程学院 西安 710049;

2. 西安交通大学机械制造系统工程国家重点实验室 西安 710054)

\begin{abstract}
摘要: 针对复杂机械系统的靶向振动控制需求, 提出不同频率分量幅值相位可控、任意频率可注入的自适应频谱塑形主动控 制算法。首先, 在传统的滤波自适应控制的结构中引入一个控制器的数字镜像模型, 通过自适应算法在线更新该数字镜像模 型的系数实现原控制器系数的随动修正, 改善了控制系统的闭环特性, 提升了控制系统的收玫性。其次, 提出了广义塑形滤 波器的设计方法, 克服了传统算法缺乏相位塑形和频率注入能力的局限性, 通过外加多频信号和噪声估计共同构造幅值、相 位和频率可控的塑形滤波器, 实现了任意频率成分的消除、消减、保持、增强、注射五种控制模式, 拓展了算法的适用性。 对所提出的算法进行了时域收玫性分析, 推导分析结果和数值仿真结果吻合良好。通过某旋转机械实测振源信号及次级通道 模型开展了算法验证，通过舱段柱壳模型开展了在线试验，结果均验证了所提出算法的有效性和优越性。
\end{abstract}

关键词: 频谱塑形; 主动控制; 振动; 自适应算法

中图分类号: TB53

\section{Adaptive Spectral Reshaping Method of Active Control for Structures}

\author{
LIU Jinxin ${ }^{1,2}$ CHEN Xuefeng ${ }^{1,2}$ ZHANG Xingwu ${ }^{1,2}$ QIAO Baijie ${ }^{1,2}$ YAN Ruqiang ${ }^{1,2}$ \\ (1. School of Mechanical Engineering, Xi'an Jiaotong University, Xi' an 710049;
}

2. State Key Laboratory for Manufacturing Systems Engineering, Xi'an Jiaotong University, Xi'an 710054)

\begin{abstract}
Aiming at the demand of targeted vibration control for complex mechanical systems, a generalized adaptive active vibration spectral reshaping control method, which is able to control amplitude and phase of every single frequency components and to inject arbitrary frequency components, is proposed. Firstly, a digital twin model (DTM) of the controller is introduced to the traditional structure of filtering adaptive control. The coefficients of the DTM are online updated according to adaptive algorithm while the original controller copies the coefficients of DTM at every iteration, which improves the convergence of the control system. Second, a design method for generalized shaping filter is proposed, overcoming the weakness of the traditional algorithm which has no phase control and frequency injection capability. External multi-frequency signal and the estimated primary noise are applied to construct the shaping filter with amplitude, phase and frequency control capability. The proposed algorithm is able to realize modes of cancellation, suppression, hold, enhancement and injection. Time domain convergence analysis of the proposed algorithm is conduct and the results are in good agreement with the numerical simulations. Experimental study has been conducted to verify the effectiveness and superiority of the proposed algorithm.
\end{abstract}

Key words: spectral reshaping; active control; vibration; adaptive algorithm

\section{0 前言}

主动控制理论和技术是振动科学和振动工程的

* 国家自然科学基金资助项目 (51705396，51911530774，52075415, 51835009)。20200119 收到初稿, 20200915 收到修改稿
重要内容。主动控制是一种利用可控振源产生振动, 用于产生振动环境或者对其他振动进行相干相消的 振动控制技术，是振动控制领域最具发展前景的研 究方向之一。主动控制因灵活性强、低频控制好、 附加质量小等优点受到学术界和工程界的广泛关 注 $^{[1-5]}$ 。经过多年的研究与积累, 主动控制在工程上 
得到广泛应用, 取得很好的效果。

无论是振动与噪声的治理还是利用, 都离不开 主动控制。通常认为主动控制对振动和噪声的消减 或消除即是治理, 主动控制对振动和噪声的产生或 增强即是利用。随着主动控制技术发展, 出现了另 一种新的控制形式: 它的控制目标既不是单一的消 减或消除, 也不是单一的产生或增强, 而是在某种 意义上二者的结合, 它希望结构的振动或噪声按照 某种预定的模式进行, 这种控制形式称为频谱塑形 主动控制。

频谱塑形主动控制可以这样定义: 它是指通过 主动控制的方法有目的地改变强干扰环境下的结构 响应频谱, 使得控制后的结构响应频谱和目标频谱 一致。频谱塑形主动控制系统在时域上表现为含有 强干扰的跟随系统。振动与噪声的消减与消除、产 生与增强以及频谱塑形三者共同构成了主动控制新 的目标和内涵, 如图 1 所示。频谱塑形主动控制最早 来源于噪声均衡 ${ }^{[6]}$, 它目前主要的应用领域为声品质 控制、心理声学、电动汽车、医学穿戴设备等 ${ }^{[7-10]}$ 。此 外, 在军事领域, 为了实现伪装和隐身, 也可以对舰 艇的振动与噪声进行频谱塑形主动控制 ${ }^{[11]}$ 。总而言之, 频谱塑形主动控制是对主动控制技术对振动工程新需 求的拓展, 目前正在蓬勃发展时期。

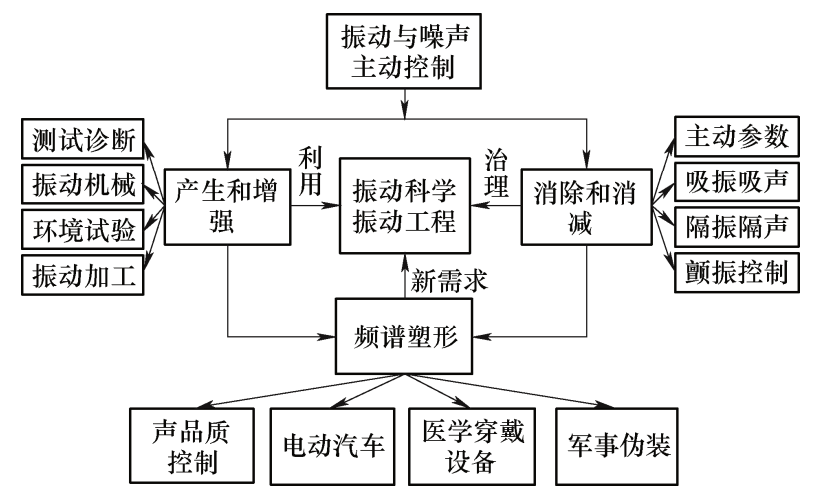

图 1 主动控制的目标与内涵

自适应频谱塑形主动控制理论是自适应主动控 制理论中发展起来的新的分支。自适应频谱塑形主 动控制最早是美国北伊利诺伊大学的 KUO 等 ${ }^{[6]}$ 于 1994 年提出。他将 FXLMS 算法中的误差信号进 行滤波, 提出了 FELMS 算法, 该算法可以通过误 差信号滤波器对残余噪声进行整形。1994 年他将 该算法拓展到窄带前馈自适应控制中, 提出了著名 的主动噪声均衡器(Active noise equalizer, ANE)算 法 $^{[12]}$ 。此后西班牙学者 DIEGO 等紧随其后进行可 多通道的 common-error ANE 算法 ${ }^{[13-14]}$ 。2005 年, KUO 等 ${ }^{[9-10,15]}$ 将其 ANE 算法引入主动声品质控制 领域, 并进行了频域算法的拓展研究。2006 年, 英
国皇家工程院院士 ELLIOTT 指出了 ANE 算法对次 级通道模型误差敏感的问题, 并提出了一种叫做调 相指令滤波 LMS 算法 ${ }^{[16]}$ 。2007 和 2008 年, 新加坡 南洋理工大学 WANG 等 ${ }^{[17-18]}$ 对主动噪声均衡器的 次级通道误差引起的均衡失配和收玫性的问题进行 了分析。2010 年, 巴西学者 OLIVEIRA 等 ${ }^{[8]}$ 提出了 归一化的 ANE 算法, 采用真实的稳态和瞬态的发动 机信号来评估控制性能。2014 年, 巴西学者 MOSQUERA-SANCHEZ 等 ${ }^{[7]}$ 提出心理声学的听觉 粗楉度指标需要对振源的相对相位进行控制, 他研 究了一种频域的幅值和相对相位控制的算法。2016 年, LIU 等 ${ }^{[11]}$ 针对 ANE 算法的均衡失配的问题, 研 究了一种增强 ANE 算法, 使得次级通道辨识存在误 差的情况下的残余噪声幅值的控制精度大大提高。

目前的频谱塑形主动控制算法大都是基于 ANE 的, 称为类 ANE 算法。类 ANE 算法有两个缺 点: 其一是它只考虑误差信号的幅值塑形, 而并不 考虑相位控制。其二是它只控制初级噪声包含的频 率成分, 而并没有频率注入(Frequency injection, FI) 的能力。但实际工程应用对相位控制和频率注入都 是有需求的。例如在水下舰艇隐身领域, 想要从频 谱塑形的角度去提升隐身性能, 就不能使得谐波相 位差成为潜在的识别特征, 同时如果频谱塑形主动 控制具有频谱注入的能力, 可以提高对探测设备的 迷惑性。因此, 希望用于水下舰艇隐身的频谱塑形 主动控制方法对线谱的幅值、相位和频率都有控制 能力, 可以为水下舰艇声隐身提供一种更一般的频 谱塑形主动控制解决方案。本文在分析 ANE 算法本 质的基础上，提出一种通用的线谱塑形主动控制方 法, 不但可以进行残余线谱振动幅值的塑形, 还可 以进行相位的塑形, 甚至可以实现频谱的注入。

\section{1 类 ANE 频谱塑形算法}

类 ANE 频谱塑形算法是基于 ANE 算法基本框 架的一类算法, 是一类针对线谱进行塑形主动控制 的方法, 它的结构如图 2 所示。假设初级振源信号 为一单频信号, 它通过系统初级通道 $P(n)$ 形成初级 噪声 $d(n)$ 。谐波生成器解析参考信号的频率, 并生 成两个单位正交的参考信号对, 即

$$
\left\{\begin{array}{l}
x_{\mathrm{c}}(n)=\cos \left(2 \pi f_{\mathrm{p}} n / f_{\mathrm{s}}\right) \\
x_{\mathrm{s}}(n)=\sin \left(2 \pi f_{\mathrm{p}} n / f_{\mathrm{s}}\right)
\end{array}\right.
$$

式中， $x_{\mathrm{c}}(n)$ 表示余弦参考信号， $x_{\mathrm{s}}(n)$ 表示余弦参 考信号, $f_{\mathrm{p}}$ 为初级振源信号频率, $n$ 表示时间序列, $f_{\mathrm{s}}$ 为采样频率。控制器输出为它们的线性组合, 即 


$$
y(n)=w_{\mathrm{c}}(n) x_{\mathrm{c}}(n)+w_{\mathrm{s}}(n) x_{\mathrm{s}}(n)
$$

式中, $w_{\mathrm{c}}(n)$ 和 $w_{\mathrm{s}}(n)$ 是控制器的系数。类 ANE 算法 中控制器的输出分为具有不同增益参数的两支, 即 消减支和均衡支。消减支的输出和均衡支的输出分 别为

$$
\left\{\begin{array}{l}
y_{\text {са }}(n)=(1-\beta) y(n) \\
y_{\text {bа }}(n)=\beta y(n)
\end{array}\right.
$$

式中, $\beta$ 是增益参数。类 $\mathrm{ANE}$ 系统的误差输出为

$$
e(n)=d(n)-y_{\mathrm{ca}}(n) * s(n)
$$

式中, $s(n)$ 是次级通道的脉冲响应函数, $d(n)$ 代表初 级噪声, *代表线性卷积。为了实现特定的残余信号 幅值, ANE 系统将一个伪误差信号反馈给自适应系 统。该伪误差信号定义为

$$
e_{\mathrm{pse}}(n)=e(n)-y_{\mathrm{ba}}(n) * \hat{s}(n)
$$

根据随机梯度法, 控制器更新方程可进一步表 示为

$$
\left\{\begin{array}{l}
w_{\mathrm{c}}(n+1)=w_{\mathrm{c}}(n)+\mu_{\mathrm{c}} e_{\mathrm{pse}}(n)\left[x_{\mathrm{c}}(n) * \hat{s}(n)\right] \\
w_{\mathrm{s}}(n+1)=w_{\mathrm{s}}(n)+\mu_{\mathrm{s}} e_{\mathrm{pse}}(n)\left[x_{\mathrm{s}}(n) * \hat{s}(n)\right]
\end{array}\right.
$$

式中, $\mu_{\mathrm{c}}$ 和 $\mu_{\mathrm{s}}$ 为迭代步长。当自适应算法收玫, 伪 误差将趋近于 0 。因此, 由式(3) (5), 令 $e_{\mathrm{pse}}(n)=0$, 残余噪声可以表示为

$$
e(n)=\beta d(n)
$$

可以看出误差 $e(n)$ 通过增益参数对初级噪声 $d(n)$ 的 幅值进行塑形。通过指定不同的增益参数, 相应的 初级噪声的频率分量可以被消除 $(\beta=0)$, 消减 $(0<\beta<$ $1)$, 保持 $(\beta=1)$ 或增强 $(\beta>1)$ 。

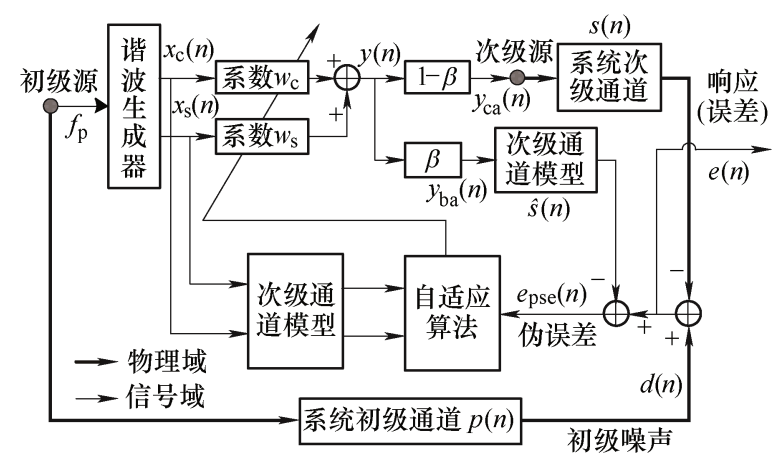

图 2 类 ANE 频谱塑形算法

\section{2 广义频谱塑形主动控制算法}

如引言所述, 类 ANE 算法尽管可以实现初级噪 声幅值塑形, 但对初级噪声小分量的塑形能力弱, 对于初级噪声不包含的分量、对相位不具备塑形能
力。因此在相位控制和频率注入的需求推动下, 研 究了一种广义的频谱塑形控制方法, 它既可以控制 幅值大小(消除、消减、保持和增强), 也可以控制 谐波间的相对相位, 而且还可以注入原来没有的频 率成分。广义频谱塑形主动控制算法是这样提出来 的：在传统的 ANE 算法中引入改进滤波 LMS(MFXLMS)结构, 获得改进 ANE(MANE)算法, 通过目标信号的设计和初级噪声信号的估计重新构 造 MANE 算法中的塑形滤波器, 即获得广义频谱塑 形主动控制算法。尽管广义频谱塑形主动控制算法 从 ANE 算法的推导而来, 但是他的最终结构已经脱 离了 ANE 算法的框架, 比 ANE 算法具有更灵活的 频谱塑形能力。下面将一步步介绍广义频谱塑形主 动控制算法的提出流程。

MFXLMS 算法由 BJARNASON 、BAO、 BRONZEL、DOELMAN、FLOCKTON 等几乎同时 在 20 世纪 90 年代初提出 ${ }^{[19]}$ 。它和传统 FXLMS 的 区别在于它通过估计初级噪声, 实现了次级通道和 控制器的交换, 大大提高了系统的收玫性。图 3 为 针对多频窄带噪声的 MFXLMS 算法, 其中 $\hat{d}(n)$ 为初 级噪声的估计, 系数可调控制器的输入和自适应算法 的输入同为滤波的参考信号, 实际工作的控制器为系 数可调控制器的镜像, 每次迭代循环他们都同步更 新。MFXLMS 算法的稳定的最大迭代步长约为 ${ }^{[19]}$

$$
\mu_{\max } \approx \frac{2}{L \cdot E\left[\hat{x}^{2}(n)\right]}
$$

而传统的 FXLMS 算法稳定的最大迭代步长约为

$$
\mu_{\max } \approx \frac{2}{(L+\Delta) \cdot E\left[\hat{x}^{2}(n)\right]}
$$

式中, $\Delta$ 为次级通道引入的纯时延, $L$ 为控制器系数 的数目, $E\left[\hat{x}^{2}(n)\right]$ 为滤波参考信号的均方值。可以 看出当次级通道纯时延较大时, MFXLMS 的收玫速 度可以远高于 FXLMS。

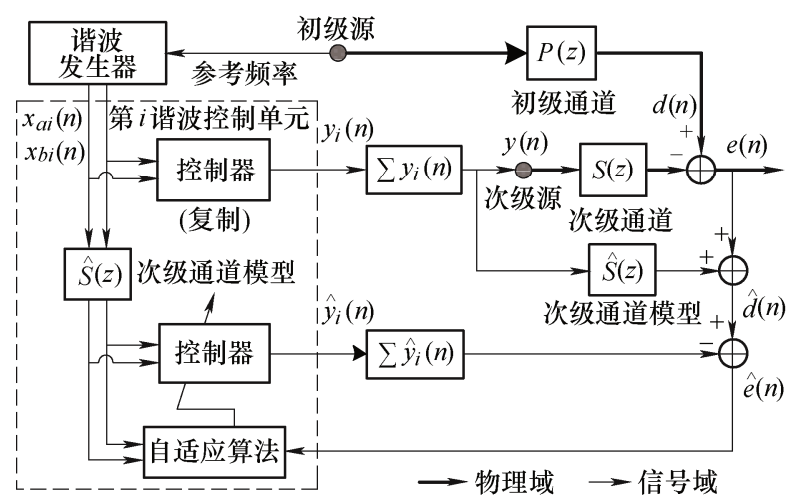

图 3 改进滤波 LMS 主动控制算法 
根据自适应频谱塑形主动控制的一般结构, 频 谱塑形主动控制算法给误差信号增加一个误差参 考, 而自适应算法使得误差信号趋近于误差参考信 号。如果可以估计出初级噪声, 并令其通过一个特 殊设计的塑形滤波器, 然后将这个塑形滤波器输出 信号作为误差参考, 则将获得初级噪声的塑形波形。 上述的 MFXLMS 也正用到初级噪声的估计, 因此 将二者合而为一, 便得改进 ANE 算法(MANE)算法。

图 4 为 MANE 的原理框图。误差参考信号为 $\hat{d}(n) * c(n)$, 其中 $c(n)$ 为塑形滤波器的脉冲响应函 数。将伪误差信号 $e_{\mathrm{pse}}(n)=\hat{d}(n) * c(n)-e(n)$ 反馈回自 适应算法, 则当伪误差信号趋近于时 0 , 误差信号将 趋近于 $\hat{d}(n) * c(n)$ 。将稳态的误差信号表示到 $z$ 域有

$$
E(z)=D(z) C(z)
$$

式中, $E(z)$ 为实际误差 $e(n)$ 的 $z$ 变换; $D(z)$ 为初级噪 声 $d(n)$ 的 $z$ 变换; $C(z)$ 塑形滤波器。可以清楚看出, MANE 的稳态控制结果就是利用 $C(z)$ 对初级噪声进 行塑形。由于塑形滤波器 $C(z)$ 的幅频特性和相频特 性是可以设计的, 因此 MANE 就具有幅值和相位的 塑形能力。

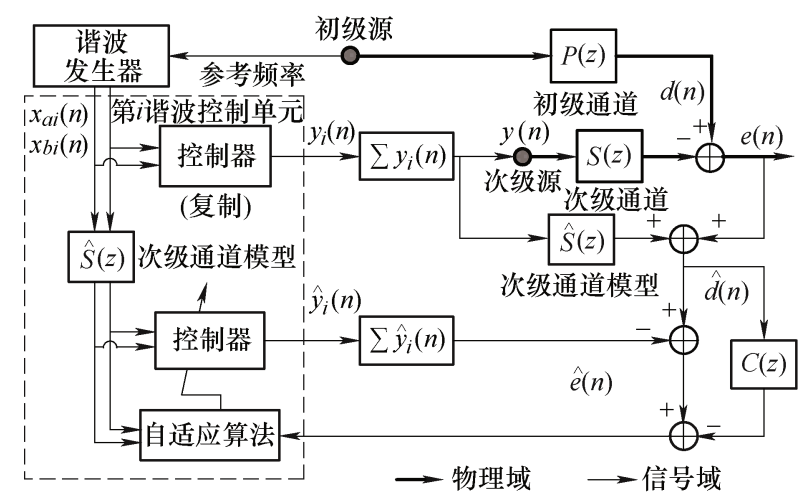

图 4 改进 ANE 频谱塑形算法

MANE 算法尽管可以实现幅值和相位的频谱 塑形主动控制, 但对于多频窄带初级噪声信号, 要 设计一个滤波器在这些频率上的相位和幅值都达到 某个预设的值并不容易。它们或者需要很长的滤波 器系数, 增加计算负担; 或者即便在目标频率处的 幅频和相频达到设计要求, 但滤波器在非目标频率 的幅频和相频将不可控的, 可能会对背景噪声产生 放大效应, 进而影响控制的效果。提出一种塑形滤 波器的设计方法, 即通过一个预设的多频信号作为 目标信号 $t(n)$, 则塑形滤波器可以定义为目标信号 和初级噪声的 $z$ 变换之比, 即

$$
C(z)=\frac{T(z)}{D(z)}
$$

式中, $T(z)$ 为预设的多频目标信号 $t(n)$ 的 $z$ 变换, $D(z)$ 为初级噪声 $d(n)$ 的 $z$ 变换。则式(10)可表示为

$$
E(z)=D(z) C(z)=T(z)
$$

具有如式(11)的塑形滤波器, 并考虑 MFXLMS 的控制结构的频谱塑形主动控制算法如图 5 所示, 可以看出它已经脱离了 ANE 的架构, 直接设计目标 信号, 即可实现灵活的频谱塑形控制。因此将该方 法称为广义频谱塑形主动控制算法。

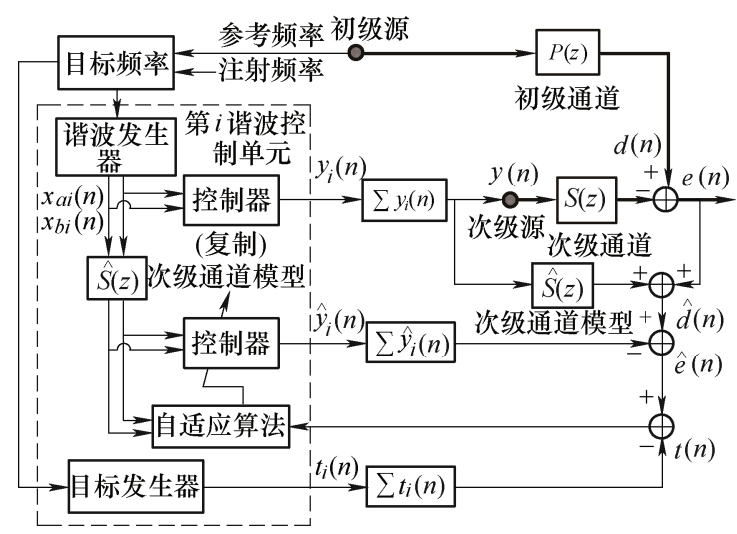

图 5 广义频谱塑形控制算法

广义频谱塑形算法的目标频率可以是任意关心 的频率点, 通常应该包含初级源的参考频率和需要 进行频率注入的注射频率

$$
\boldsymbol{f}_{\mathrm{t}}=\left(\begin{array}{ll}
\boldsymbol{f}_{\mathrm{p}}^{\mathrm{T}} & \boldsymbol{f}_{\text {inj }}^{\mathrm{T}}
\end{array}\right)^{\mathrm{T}}
$$

式中, $\boldsymbol{f}_{\mathrm{p}}^{\mathrm{T}}$ 为初级噪声频率向量, $\boldsymbol{f}_{\mathrm{inj}}^{\mathrm{T}}$ 为注入噪声频 率向量, 目标频率数目是初级噪声频率和注入噪声 频率数目之和。可见目标频率向量由初级频率向量 和注入频率向量串联而成, 长度是二者长度之和。 次级通道在不同目标频率会引入不同的幅值比和相 位差, 定义为

$$
\left\{\begin{array}{l}
\boldsymbol{p}_{\mathrm{s}}=\left\{\left|S\left(2 \pi f_{i} n / f_{\mathrm{s}}\right)\right|\right\}^{\mathrm{T}} \\
\boldsymbol{\theta}_{\mathrm{s}}=\left\{\angle S\left(2 \pi f_{i} n / f_{\mathrm{s}}\right)\right\}^{\mathrm{T}}
\end{array}\right.
$$

对于多频频谱塑形主动控制中, 需要引入幅值 修正使得不同频率具有相近的收敛速度。具有幅值 修正的参考信号向量对可表示为

$$
\boldsymbol{P}_{\text {rec }}=\operatorname{diag}\left[\boldsymbol{p}_{\text {rec }}\right], \boldsymbol{p}_{\text {rec }}=\max \left[\boldsymbol{p}_{\mathrm{s}}\right] \boldsymbol{p}_{\mathrm{s}}^{-1}
$$

式中, $\boldsymbol{P}_{\mathrm{rec}}$ 为包含幅值修正系数的对角矩阵, 下标 rec 表示修正。广义频谱塑形算法的参考信号向量根 据目标频率向量生成, 即

$$
\left\{\begin{array}{l}
\boldsymbol{x}_{\mathrm{c}}(n)=\boldsymbol{P}_{\mathrm{rec}} \cos \left(2 \pi f_{\mathrm{t}} n / f_{\mathrm{s}}\right) \\
\boldsymbol{x}_{\mathrm{s}}(n)=\boldsymbol{P}_{\mathrm{rec}} \sin \left(2 \pi f_{\mathrm{t}} n / f_{\mathrm{s}}\right)
\end{array}\right.
$$

控制器输出可以表达为 


$$
y(n)=\boldsymbol{w}_{\mathrm{c}}^{\mathrm{T}}(n) \boldsymbol{x}_{\mathrm{c}}(n)+\boldsymbol{w}_{\mathrm{s}}^{\mathrm{T}}(n) \boldsymbol{x}_{\mathrm{s}}(n)
$$

式中, $\boldsymbol{w}_{\mathrm{c}}(n)$ 和 $\boldsymbol{w}_{\mathrm{s}}(n)$ 是控制器的系数向量。误差 为

$$
e(n)=d(n)-y(n) * s(n)
$$

估计的噪声为

$$
\hat{d}(n)=e(n)+y(n) * \hat{s}(n)
$$

估计的误差信号为

$$
\begin{gathered}
\hat{e}(n)=\hat{d}(n)-\boldsymbol{w}_{\mathrm{c}}^{\mathrm{T}}(n)\left[\boldsymbol{x}_{\mathrm{c}}(n) * \hat{s}(n)\right]- \\
\boldsymbol{w}_{\mathrm{s}}^{\mathrm{T}}(n)\left[\boldsymbol{x}_{\mathrm{s}}(n) * \hat{s}(n)\right]
\end{gathered}
$$

伪误差信号为

$$
e_{\mathrm{pse}}(n)=\hat{e}(n)-t(n)
$$

由式(18)和(19) 可知, 如果 $\hat{s}(n)=s(n)$, 则估计的初 级噪声等于真实的, 即 $\hat{d}(n)=d(n)$ 。考虑缓慢更新 权值, 控制器和次级通道的位置可以交换, 因此式 (20)可以进一步表达为

$$
\hat{e}(n)=\hat{d}(n)-\left[\boldsymbol{w}_{\mathrm{c}}^{\mathrm{T}}(n) \boldsymbol{x}_{\mathrm{c}}(n)+\boldsymbol{w}_{\mathrm{s}}^{\mathrm{T}}(n) \boldsymbol{x}_{\mathrm{s}}(n)\right] * \hat{s}(n)
$$

将式(17)、(19)代入式(22), 则有 $\hat{e}(n)=e(n)$ 。因此, 式(21)中的伪误差信号可以表达为

$$
e_{\mathrm{pse}}(n)=e(n)-t(n)
$$

控制器更新是基于 FXLMS 方法, 因此有

$$
\left\{\begin{array}{l}
\boldsymbol{w}_{\mathrm{c}}(n+1)=\boldsymbol{w}_{\mathrm{c}}(n)+\mu_{\mathrm{c}} e_{\mathrm{pse}}(n)\left[\boldsymbol{x}_{\mathrm{c}}(n) * \hat{s}(n)\right] \\
\boldsymbol{w}_{\mathrm{s}}(n+1)=\boldsymbol{w}_{\mathrm{s}}(n)+\mu_{\mathrm{s}} e_{\mathrm{pse}}(n)\left[\boldsymbol{x}_{\mathrm{s}}(n) * \hat{s}(n)\right]
\end{array}\right.
$$

式中, $\mu_{\mathrm{c}}$ 和 $\mu_{\mathrm{s}}$ 为迭代步长。当算法收敛时, $e_{\mathrm{pse}}(n)$ 趋近于 0 。因此, 由式(23)可知, 稳态误差为

$$
e(n)=t(n)
$$

由于 $t(n)$ 的幅值、相位和频率都预定义的多频 信号, 因此系统响应的幅值、相位和频率均可得到 控制。

\section{3 算法性能分析}

广义频谱塑形主动控制算法的收敛性可以在时 域进行分析。首先由式(20)和(23)可知, 伪误差信号 表达为

$$
e_{\mathrm{pse}}(n)=d(n)-t(n)-\left[\boldsymbol{w}_{\mathrm{c}}^{\mathrm{T}}(n) \boldsymbol{x}_{\mathrm{c}, \mathrm{f}}(n)+\boldsymbol{w}_{\mathrm{s}}^{\mathrm{T}}(n) \boldsymbol{x}_{\mathrm{s}, \mathrm{f}}(n)\right]
$$

式中, $\boldsymbol{x}_{\mathrm{c}, \mathrm{f}}(n)$ 和 $\boldsymbol{x}_{\mathrm{s}, \mathrm{f}}(n)$ 为滤波的参考信号。对于周 期信号, 次级通道将引入幅值比和相位差(式(14))。 因此滤波的参考信号表达为

$$
\left\{\begin{array}{l}
\boldsymbol{x}_{\mathrm{c}, \mathrm{f}}(n)=p_{\mathrm{s}, \max }\left[\cos \boldsymbol{\Theta}_{\mathrm{s}} \cdot \boldsymbol{x}_{\mathrm{c}}(n)-\sin \boldsymbol{\Theta}_{\mathrm{s}} \cdot \boldsymbol{x}_{\mathrm{c}}(n)\right] \\
\boldsymbol{x}_{\mathrm{s}, \mathrm{f}}(n)=p_{\mathrm{s}, \max }\left[\cos \boldsymbol{\Theta}_{\mathrm{s}} \cdot \boldsymbol{x}_{\mathrm{s}}(n)+\sin \boldsymbol{\Theta}_{\mathrm{s}} \cdot \boldsymbol{x}_{\mathrm{s}}(n)\right]
\end{array}\right.
$$

式中, $\cos \boldsymbol{\Theta}_{\mathrm{s}}$ 定义为次级通道相引入位差的余弦为 对角元的矩阵, $\sin \Theta_{\mathrm{s}}$ 定义为次级通道相引入位差的 正弦为对角元的矩阵, $p_{\mathrm{s}, \max }$ 定义为次级通道将引入 幅值比的最大值，即

$$
\left\{\begin{array}{l}
\cos \boldsymbol{\Theta}_{\mathrm{s}} \equiv \operatorname{diag}\left[\cos \boldsymbol{\theta}_{\mathrm{s}}\right] \\
\sin \boldsymbol{\Theta}_{\mathrm{s}} \equiv \operatorname{diag}\left[\sin \boldsymbol{\theta}_{\mathrm{s}}\right] \\
p_{\mathrm{s}, \max }=\max \left[\boldsymbol{p}_{\mathrm{s}}\right]
\end{array}\right.
$$

将式(27)代入式(26), 并令

$$
\begin{array}{r}
\left\{\begin{array}{l}
d(n)=\boldsymbol{a}_{\mathrm{p}} \cdot \boldsymbol{x}_{\mathrm{c}}(n)+\boldsymbol{b}_{\mathrm{p}} \cdot \boldsymbol{x}_{\mathrm{s}}(n) \\
t(n)=\boldsymbol{a}_{\mathrm{t}} \cdot \boldsymbol{x}_{\mathrm{c}}(n)+\boldsymbol{b}_{\mathrm{t}} \cdot \boldsymbol{x}_{\mathrm{s}}(n)
\end{array}\right. \\
\left\{\begin{array}{l}
\boldsymbol{a}=\boldsymbol{a}_{\mathrm{p}}-\boldsymbol{a}_{\mathrm{t}} \\
\boldsymbol{b}=\boldsymbol{b}_{\mathrm{p}}-\boldsymbol{b}_{\mathrm{t}}
\end{array}\right.
\end{array}
$$

伪误差信号可以进一步表达为

$$
\begin{array}{r}
e_{\mathrm{pse}}(n)=\left[\boldsymbol{a}-\boldsymbol{w}_{\mathrm{c}, \mathrm{f}}(n)\right]^{\mathrm{T}} \boldsymbol{x}_{\mathrm{c}}(n)+\left[\boldsymbol{b}-\boldsymbol{w}_{\mathrm{s}, \mathrm{f}}(n)\right]^{\mathrm{T}} \boldsymbol{x}_{\mathrm{s}}(n) \\
\left\{\begin{array}{l}
\boldsymbol{w}_{\mathrm{c}, \mathrm{f}}(n)=p_{\mathrm{s}, \max }\left[\cos \boldsymbol{\Theta}_{s} \cdot \boldsymbol{w}_{\mathrm{c}}(n)+\sin \boldsymbol{\Theta}_{s} \cdot \boldsymbol{w}_{\mathrm{s}}(n)\right] \\
\boldsymbol{w}_{\mathrm{s}, \mathrm{f}}(n)=p_{\mathrm{s}, \max }\left[\cos \boldsymbol{\Theta}_{s} \cdot \boldsymbol{w}_{\mathrm{s}}(n)-\sin \boldsymbol{\Theta}_{s} \cdot \boldsymbol{w}_{\mathrm{c}}(n)\right]
\end{array}\right.
\end{array}
$$

从式(31)可知, 伪误差信号的期望为

$$
\begin{gathered}
E\left[e_{\mathrm{pse}}^{2}(n)\right]=\left[\boldsymbol{a}-\boldsymbol{w}_{\mathrm{c}, \mathrm{f}}(n)\right]^{\mathrm{T}} \boldsymbol{P}_{\mathrm{s}}\left[\boldsymbol{a}-\boldsymbol{w}_{\mathrm{c}, \mathrm{f}}(n)\right]+ \\
{\left[\boldsymbol{b}-\boldsymbol{w}_{\mathrm{s}, \mathrm{f}}(n)\right]^{\mathrm{T}} \boldsymbol{P}_{\mathrm{c}}\left[\boldsymbol{b}-\boldsymbol{w}_{\mathrm{s}, \mathrm{f}}(n)\right]} \\
\boldsymbol{P}_{l}=E\left[\boldsymbol{x}_{l}(n) \boldsymbol{x}_{l}^{\mathrm{T}}(n)\right] \quad(l=\mathrm{c}, \quad \mathrm{s})
\end{gathered}
$$

控制器系数调整根据随机梯度法, 有

$$
\boldsymbol{w}_{l}(n+1)=\boldsymbol{w}_{l}(n)+\frac{\mu_{l}}{2} \frac{\partial E\left[e_{\mathrm{pse}}^{2}(n)\right]}{\partial \boldsymbol{w}_{l}(n)} \quad(l=\mathrm{c}, \mathrm{s})
$$

式中, 梯度可以通过将式(32)代入式(33), 再代入式 (35), 系数更新公式可以进一步表达为

$$
\boldsymbol{w}_{l}(n+1)=\boldsymbol{K}_{l} \boldsymbol{w}_{l}(n)+\boldsymbol{m}_{l} \quad(l=\mathrm{c}, \mathrm{s})
$$

$$
\left\{\begin{array}{l}
\boldsymbol{K}_{l}=\boldsymbol{I}-\mu_{l} p_{\mathrm{s}, \max }^{2} \boldsymbol{P}_{x, l} \quad(l=\mathrm{c}, \quad \mathrm{s}) \\
\boldsymbol{m}_{\mathrm{c}}=\mu_{\mathrm{c}} p_{\mathrm{s}, \max } \boldsymbol{P}_{\mathrm{c}}\left[\cos \boldsymbol{\Theta}_{\mathrm{s}} \cdot \boldsymbol{a}-\sin \boldsymbol{\Theta}_{\mathrm{s}} \cdot \boldsymbol{b}\right] \\
\boldsymbol{m}_{\mathrm{s}}=\mu_{\mathrm{s}} p_{\mathrm{s}, \max } \boldsymbol{P}_{\mathrm{s}}\left[\sin \boldsymbol{\Theta}_{\mathrm{s}} \cdot \boldsymbol{a}+\cos \boldsymbol{\Theta}_{\mathrm{s}} \cdot \boldsymbol{b}\right]
\end{array}\right.
$$

系数收敛方程为

$\boldsymbol{w}_{l}(n)=\boldsymbol{K}_{l}^{n} \boldsymbol{w}_{l}(0)+\left(\boldsymbol{I}-\boldsymbol{K}_{l}\right)^{-1}\left(\boldsymbol{I}-\boldsymbol{K}_{l}^{n-1}\right) \boldsymbol{m}_{l}(l=\mathrm{c}, \mathrm{s})$

根据式(38), 如果 $\left\|\boldsymbol{K}_{l}\right\|_{\infty}<1$, 控制器系数的稳态值为

$$
\left\{\begin{array}{l}
\boldsymbol{w}_{\mathrm{c}}(\infty)=\left(\boldsymbol{I}-\boldsymbol{K}_{\mathrm{c}}\right)^{-1} \boldsymbol{m}_{\mathrm{c}}=p_{\mathrm{s}, \max }^{-1}\left[\cos \boldsymbol{\Theta}_{\mathrm{s}} \cdot \boldsymbol{a}-\sin \boldsymbol{\Theta}_{\mathrm{s}} \cdot \boldsymbol{b}\right] \\
\boldsymbol{w}_{\mathrm{s}}(\infty)=\left(\boldsymbol{I}-\boldsymbol{K}_{\mathrm{s}}\right)^{-1} \boldsymbol{m}_{\mathrm{s}}=p_{\mathrm{s}, \max }^{-1}\left[\sin \boldsymbol{\Theta}_{\mathrm{s}} \cdot \boldsymbol{a}+\cos \boldsymbol{\Theta}_{\mathrm{s}} \cdot \boldsymbol{b}\right]
\end{array}\right.
$$


收敛步长为

$$
0<\mu_{l}<\frac{2}{p_{\mathrm{s}, \max }^{2}\left\|\boldsymbol{P}_{l}\right\|_{\infty}} \quad(l=\mathrm{c}, \mathrm{s})
$$

由式(16)、(34)得 $\left\|\boldsymbol{P}_{l}\right\|_{\infty}=0.5$ 。实际误差信号的实时 幅值和相位为

$$
\left\{\begin{array}{l}
\boldsymbol{p}_{\mathrm{e}}(n)=\sqrt{\left[\boldsymbol{a}_{\mathrm{p}}-\boldsymbol{w}_{\mathrm{c}, \mathrm{f}}(n)\right]^{2}+\left[\boldsymbol{b}_{\mathrm{p}}-\boldsymbol{w}_{\mathrm{s}, \mathrm{f}}(n)\right]^{2}} \\
\boldsymbol{\theta}_{\mathrm{e}}(n)=\arctan \frac{\boldsymbol{b}_{\mathrm{p}}-\boldsymbol{w}_{\mathrm{s}, \mathrm{f}}(n)}{\boldsymbol{a}_{\mathrm{p}}-\boldsymbol{w}_{\mathrm{c}, \mathrm{f}}(n)}
\end{array}\right.
$$

实际误差信号的稳态幅值和相位为

$$
\left\{\begin{array}{l}
\boldsymbol{p}_{\mathrm{e}}(\infty)=\sqrt{\boldsymbol{a}_{\mathrm{t}}{ }^{2}+\boldsymbol{b}_{\mathrm{t}}{ }^{2}} \\
\boldsymbol{\theta}_{\mathrm{e}}(\infty)=\arctan \frac{\boldsymbol{b}_{\mathrm{t}}}{\boldsymbol{a}_{\mathrm{t}}}
\end{array}\right.
$$

可见理论稳态幅值和相位等于目标信号的幅值 和相位。通过一组仿真来验证推到的准确性。初 级噪声包含 $10 \mathrm{~Hz}$ 和 $50 \mathrm{~Hz}$ 两个分量, 幅值均为 $1 \mathrm{~dB}$, 初始相位为 $45^{\circ}$ 和 $0^{\circ}$, 目标幅值设定为 $0.5 \mathrm{~dB}$, 目标相位设定为 $0^{\circ}, 30^{\circ}$, 采样频率 1024 $\mathrm{Hz}$, 次级通道离散模型为

$$
S(z)=0.01 \times \frac{z^{-1}+0.99 z^{-2}}{1-1.95 z^{-2}+0.98 z^{-2}}
$$

误差信号的实时幅值和相位如图 6 所示, 其中 理论曲线通过式(41)计算, 仿真曲线是通过离散傅 里叶变换在残余信号中提取。从图 6 可以看出理论 和仿真的结果吻合很好。

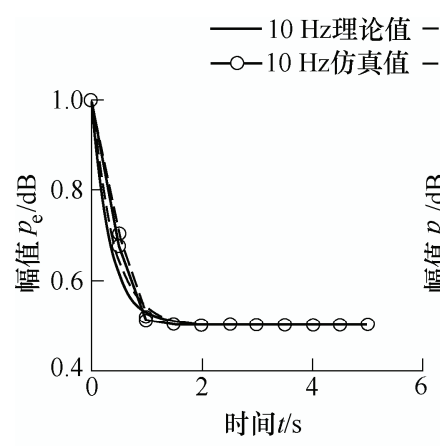

(a)

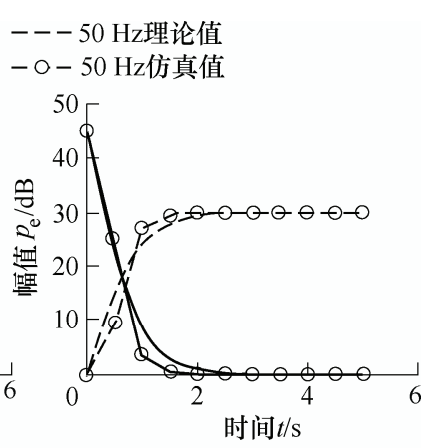

(b)
图 6 误差信号幅值和相位的理论和仿真比较

\section{4 实测数据验证}

在实测数据验证算例中, 考虑从某旋转机械振 源测量的初级噪声和实际结构测量的次级通道模型 进行算法验证。图 7 显示了采集的初级噪声的时域 信号及频域信号, 可以看出它为典型的多频叠加宽 带噪声信号。采样频率设置为 $3000 \mathrm{~Hz}$, 采样时间
为 $6.83 \mathrm{~s}$ 。对该信号进行初步分析, 可以获得它的 各阶频率分别为 $38.6 \mathrm{~Hz}(1 \times) 、 77.2 \mathrm{~Hz}(2 \times) 、 115.8$ $\mathrm{Hz}(3 \times) 、 154.4 \mathrm{~Hz}(4 \times)$ 和 $193.1 \mathrm{~Hz}(5 \times)$ 。其中各个分 量的幅值分别约为 $0.0087 g 、 0.0113 g 、 0.0034 g$ 、 $0.0019 \mathrm{~g}$ 和 $0.0012 \mathrm{~g}$ 。各个频率的初始相位分别约为 $88.00^{\circ} 、 57.63^{\circ} 、 89.81^{\circ} 、 111.1^{\circ}$ 和 $154.3^{\circ}$ 。实 测数据辨识的频响函数如图 8 所示, 其中辨识模型 采用了 80 个前项系数和 80 个反向系数的 IIR 滤波 器。下面将基于上述的初级噪声的次级通道模型进 行两个算例研究。

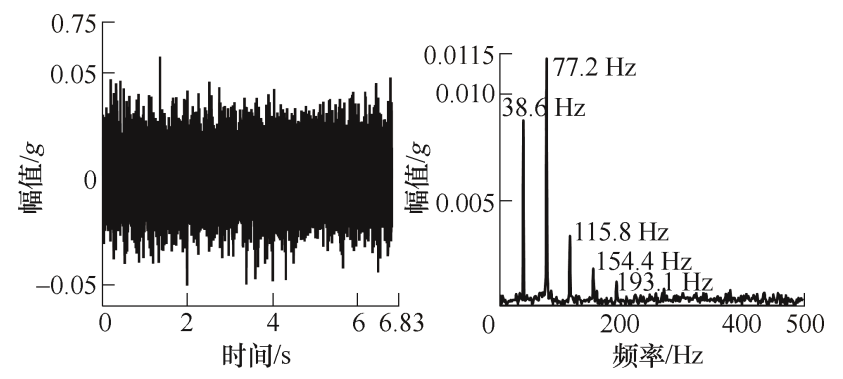

图 7 转子试验台的实测初级噪声
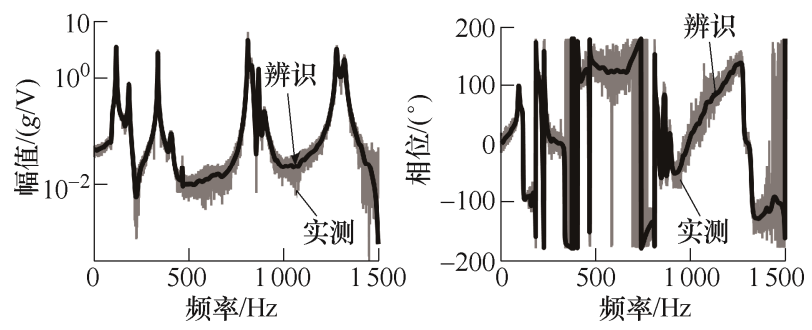

图 8 结构实测次级通道频响函数

\section{1 验证算例 1}

在验证算例 1 中, 主要关注广义频谱塑形主动 控制算法的相位控制能力及频率注入能力。因此, 将所有的幅值比都设为 1 , 即期望响应的所有频率 的幅值都不改变。特别的对于注入模式, 设置增益 参数为 0.6 , 表示期望的目标幅值为基频 $(1 \times)$ 的 $60 \%$ 。 每个分量的目标相位分别为 $10^{\circ} 、 30^{\circ} 、 50^{\circ}$ 、 $70^{\circ} 、 90^{\circ} 、 160^{\circ}$ 。控制结果如图 9 所示。其中图 $9 \mathrm{a}$ 上图和下图均表示有控制和无控制的时候的误差 信号的对比，它们的区别是表示的时段不同，上图表 示 $0 \sim 0.1 \mathrm{~s}$, 下图表示 $6.6 \sim 6.7 \mathrm{~s}$ 。可以看出, 有控制 和没控制的误差信号在刚开始的时候相等, 过了一段 时间后，有控制的误差信号相位发生了偏移，但幅值 大致上保持不变。图 $9 b$ 展示了有控制和无控制下误差 信号的稳态频谱图。可进一步看出: (1) $1 \times$ 到 $5 \times$ 分量 的幅值都保持不变; (2) 有一个 $231.65 \mathrm{~Hz}$ 分量的注入。 图9c、9d分别显示了每个分量幅值和相位的收敛趋势。 可以看出：(1) 每个分量的幅值都保持在虚线附近不 变; (2) 相位趋近于虚线所示的目标相位。 


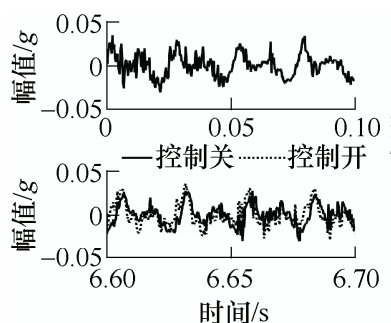

(a) (c)

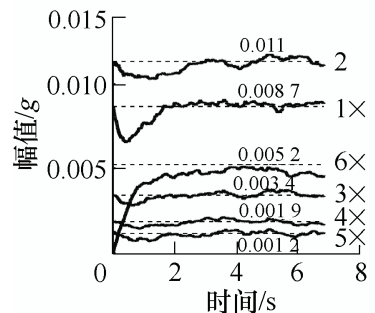

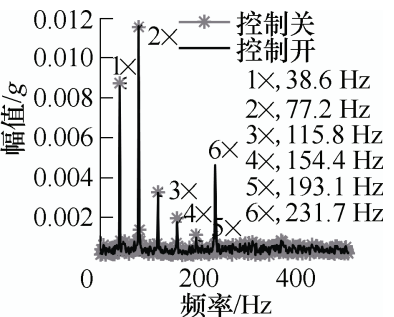

(b)

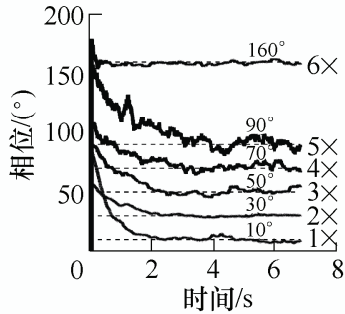

(d)
图 9 频谱塑形控制验证算例 1

\section{2 验证算例 2}

在验证算例 2 中, 考虑更加一般的频谱塑形模 式, 即消除、消减、保持、增强、注射的五种工作 模式。为 $1 \times$ 到 $6 \times$ 分别设置的增益参数为 $0 、 0.6 、 1$ 、 $3 、 4$ 和 0.9 , 分别代表消除 $1 \times$ 分量, 消减 $2 \times$ 分量为 其 $60 \%$ ，保持 $3 \times$ 分量，增强 $4 \times$ 为其 $200 \%$ ，增强 $5 \times$ 分量为其 $400 \%$, 注入 $6 \times$ 分量, 其中幅值为基频 $(1 \times)$ 的 $90 \%$ 。各个分量的相位定义为 $0^{\circ} 、 160^{\circ} 、 130^{\circ}$ 、 $100^{\circ} 、 70^{\circ} 、 40^{\circ}$ 。图 10 显示了控制的结果。其中 所有子图代表的意义和算例 1 一致。从图 10a 可以 看出有控制和无控制的误差信号开始的时候相等, 一段时间过后，有控制的误差信号的相位和幅值(波 形的形状)都发生了改变。图 $10 \mathrm{~b}$ 给出了一个更加清 晰的展示, 可以看出五种工作模式(消除、消减、保 持、增强和注入)成功实现。图 10c、10d 显示了误差 信号幅值和相位的收玫趋势, 可以看出它们也都可以 准确地收玫于它们的目标(虚线), 包括注入频率。

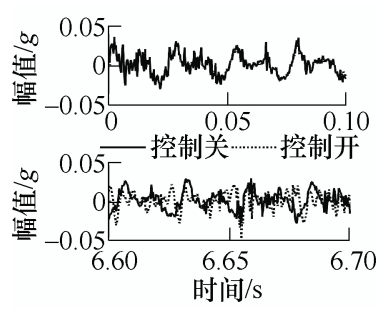

(a)

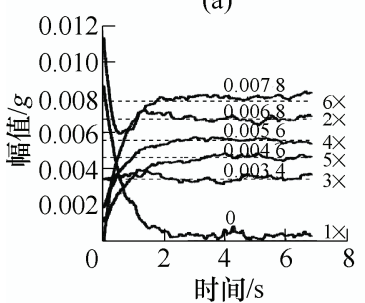

(c)

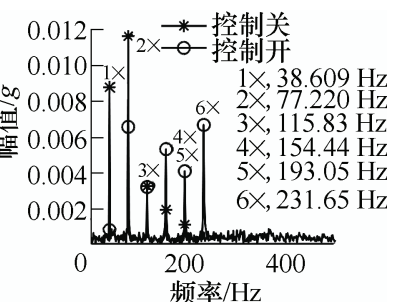

(b)

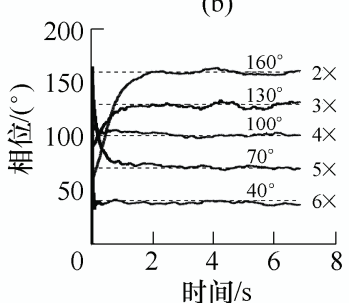

(d)
图 10 频谱塑形控制验证算例 2

\section{5 在线试验验证}

在线试验验证的系统为一个四舱段柱壳模型, 如图 11 所示。其中振源采用偏心电机进行振动的模 拟。舱体直接采用螺栓连接, 便于内部结构安装, 舱体之间采用阻尼隔断。舱体振源置于弹簧隔振器 之上模拟被动隔振。试验台通过支架安装于 $\mathrm{T}$ 型台 基座。控制系统采用的仪器为 NI 的实时控制器, 整个系统包含计算机、IEPE 调理器(联能 YE3821)、 NI 实时控制器(NI PXIe-8115)、NI FPGA 模块(NI PXI-7853R)、FPGA 接线盒、ModalShop 激振器和 PCB 加速度传感器等。次级通道辨识结, 采用的滤 波器阶数为 $(50,50)$, 采样频率为 $2048 \mathrm{~Hz}$ 。

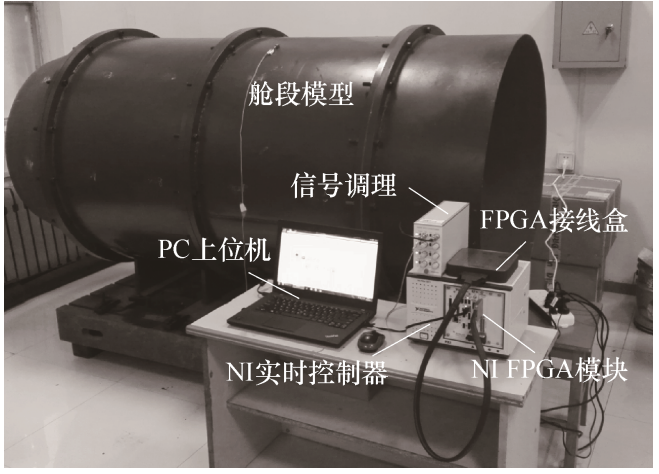

图 11 试验验证的舱段模型

在线主动控制试验和系统辨识采用相同的仪器 以及相同的作动器布置, 移除系统辨识中的振源模 拟激振器, 采用小型偏心电机模拟初级振源。振源 的基频为 $47.88 \mathrm{~Hz}$, 分别控制前 4 阶谐波, 即 $47.88 \mathrm{~Hz} 、 95.76 \mathrm{~Hz} 、 143.64 \mathrm{~Hz}$ 和 $191.52 \mathrm{~Hz}$ 。此外, 在频率注入的应用中还考虑第 5 阶谐波，即 $239.4 \mathrm{~Hz}$ 。图 12 为采用广义频谱塑形主动控制算法 的主动减振模式的误差信号的时域图和频域图。可 以看出, 误差信号的幅值在时域上获得了衰减，在 频域上前 4 阶谐波成功被消除。图 13 为采用广义 频谱塑形主动控制算法的频谱塑形模式的误差信号 的时域图和频域图。可以看出，误差在时域上某些 时刻有放大作用，在频域上第 $1 、 3$ 和 4 阶谐波被
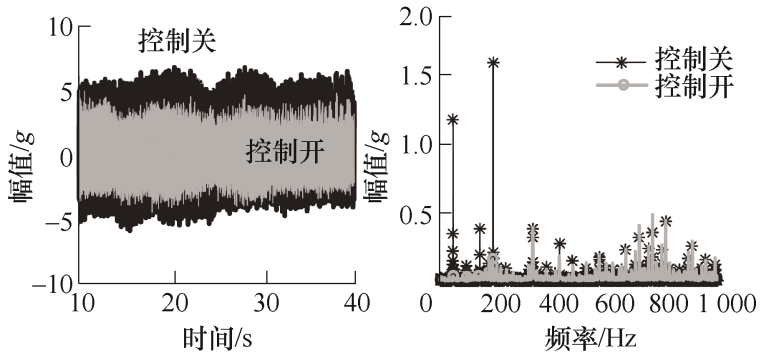

图 12 频谱塑形控制算法的主动减振模式 
成功消除, 第阶 2 谐波被增强, 第 5 阶谐波处做了 频率注入。总而言之, 所提出方法成功地实现了频 谱塑形主动控制和主动减振控制。
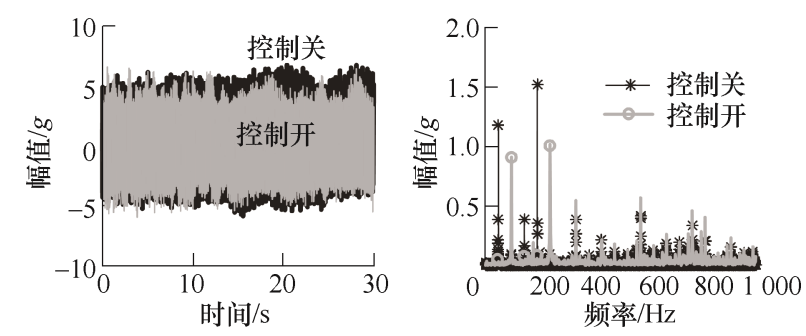

图 13 频谱塑形算法的频谱塑形模式

\section{6 结论}

论文首先分析并指出了类 ANE 算法缺乏相位 塑形和频率注入能力的局限性。它通过初级噪声的 估计来形成伪误差信号, 其中增益参数只对初级噪 声估计进行幅值的缩放, 而保持相位不变, 因此它 并无相位塑形能力。由于误差参考信号来自于初级 噪声的估计, 并不包含初级噪声之外的频率分量, 因此 ANE 算法也并没有注入频率的能力。总而言 之, 相位塑形和频率注入的能力是频谱塑形主动控 制中不可或缺的重要能力, 而目前的频谱塑形主动 控制算法正缺乏这样的能力。

其次, 提出了幅值相位可控、任意频率可注入 的广义频谱塑形主动控制算法。克服了传统算法缺 乏相位塑形和频率注入能力的局限性, 实现了任意 频率成分的消除、消减、保持、增强、注射五种控 制模式。分别通过实测信号以及舱段柱壳模型进行 了试验, 验证了所提出算法的有效性和优越性。 综上所述, 所提出的广义频谱塑形主动方法可以 进行幅值和相位的塑形, 并且可以根据需求进行 频率注入, 是一种更完善的频谱塑形主动控制解决 方案。

\section{参 考 文 献}

[1] 黄文虎, 曹登庆, 韩增尧. 航天器动力学与控制的研究 进展与展望[J]. 力学进展, 2012, 42(4): 367-394.

HUANG Wenhu, CAO Dengqing, HAN Zengyao. Adcances and trends in dynamics and control of spacecrafts[J]. Advances in Mechanics, 2012, 42(4): 367-394.

[2] 杨铁军, 靳国永, 刘志刚. 船舶动力装置振动的主动控 制[M]. 哈尔滨: 哈尔滨工程大学出版社, 2011.

YANG Tiejun, JIN Guoyong, LIU Zhigang. Active vibration control of ship power installation[M]. Harbin: Harbin Engineering University Press, 2011.
[3] 朱石坚, 何琳. 舰船水声隐身技术 (二)[J]. 噪声与振动 控制, 2002(4): 12-15.

ZHU Shijian, HE Lin. Warships water-borne noise concealment techniques(2)[J]. Noise and Vibration Control, 2002(4): 12-15.

[4] ZECH P, PLÖGER D F, RINDERKNECHT S. Active control of planetary gearbox vibration using phase-exact and narrowband simultaneous equations adaptation without explicitly identified secondary path models[J]. Mechanical Systems and Signal Processing, 2019(120): 234-251.

[5] 丁庆中, 李以农, 张锋, 等. 基于自适应滤波算法的齿 轮传动系统振动主动控制 [J]. 机械工程学报, 2013, 49(15): 74-81.

DING Qingzhong, LI Yinong, ZHANG Feng, et al. Adaptive filter algorithm for active vibration control of gear transmission [J]. Journal of Mechanical Engineering, 2013, 49(15): 74-81.

[6] KUO S M, TSAI J. Residual noise shaping technique for active noise control systems[J]. Journal of the Acoustical Society of America, 1994, 95: 1665-1668.

[7] MOSQUERA-SANCHEZ J A, DE OLIVEIRA L P R. A multi-harmonic amplitude and relative-phase controller for active sound quality control[J]. Mechanical Systems and Signal Processing, 2014, 45(2): 542-562.

[8] OLIVEIRA L P R, STALLAERT B, JANSSENS K, et al. NEX-LMS : A novel adaptive control scheme for harmonic sound quality control[J]. Mechanical Systems and Signal Processing, 2010, 24(6): 1727-1738.

[9] KUO $\mathrm{S}$ M, YENDURI $\mathrm{R}$ K, GUPTA A. Frequency-domain delayless active sound quality control algorithm[J]. Journal of Sound and Vibration, 2008, 318(4-5): 715-724.

[10] KUO S M, GUPTA A, MALLU S. Development of adaptive algorithm for active sound quality control[J]. Journal of Sound and Vibration, 2007, 299(1-2): 12-21.

[11] LIU J, CHEN X. Adaptive compensation of misequalization in narrowband active noise equalizer systems[J]. IEEE/ACM Transactions on Audio, Speech, and Language Processing, 2016, 24(12): 2390-2399.

[12] KUO S M, JI M J. Principle and application of adaptive noise equalizer[J]. Circuits \& Systems II Analog \& Digital Signal, 1994, 41(7): 471-474.

[13] GONZALEZ A, DE DIEGO M, FERRER M, et al. Multichannel active noise equalization of interior noise[J]. IEEE Transactions on Audio Speech and Language Processing, 2006, 14(1): 110-122.

[14] DIEGO M D, GONZALEZ A, FERRER M, et al. (下转第 106 页) 
[17] WANG L, XIONG Y, WANG Z, et al. Temporal segment networks : Towards good practices for deep action recognition[C]//Proceedings of the European Conference on Computer Vision, 2016: 20-36.

[18] QIU Z, YAO T, MEI T. Learning spatio-temporal representation with pseudo-3d residual networks[C]// Proceedings of the IEEE International Conference on Computer Vision, 2017: 5534-5542.

[19] CARREIRA J, ZISSERMAN A. Quo vadis, action recognition? A new model and the kinetics dataset $[\mathrm{C} / \mathrm{CD}] / /$ Proceedings of the IEEE Conference on
Computer Vision and Pattern Recognition, 2017.

[20] FEICHTENHOFER C, FAN H, MALIK J, et al. SlowFast networks for video recognition[C]// International Conference on Computer Vision, 2018: 6201-6210.

作者简介: 邓堡元, 男, 1996 年出生, 博士研究生。主要研究方向为红 外机器视觉、红外检测。

E-mail: dengbaoyuan@foxmail.com

何望泽(通信作者), 男, 1983 年出生, 副教授。主要研究方向为红外机 器视觉、红外无损检测、电磁无损检测、声发射检测。

E-mail: yhe@vip.163.com

\section{(上接第 97 页)}

Multichannel active noise control system for local spectral reshaping of multifrequency noise[J]. Journal of Sound and Vibration, 2004, 274: 249-271.

[15] KUO S M, MALLU S. Adaptive active sound quality control algorithm $[\mathrm{C}] /$ Proceedings of International Symposium on Intelligent Signal Processing and Communication Systems 2005 (ISPACS 2005): 2005: 737-740.

[16] REES L E, ELLIOTT S J. Adaptive algorithms for active sound-profiling $[\mathrm{J}]$. IEEE Transactions On Audio Speech and Language Processing, 2006, 14(2): 711-719.

[17] WANG L, GAN W S. Convergence analysis of narrowband active noise equalizer system under imperfect secondary path estimation[J]. IEEE Transactions On
Audio Speech and Language Processing, 2009, 17(4): 566-571.

[18] WANG L, GAN W S. Analysis of misequalization in a narrowband active noise equalizer system[J]. Journal of Sound and Vibration, 2008, 311(3-5): 1438-1446.

[19] ELLIOTT S. Signal processing for active control[M]. London: Academic Press, 2000.

作者简介: 刘金金, 男, 1988 年出生, 博士, 副教授, 博士研究生导师。 主要研究方向为机械动力学振动控制、发动机主动安全控制、机器人智 能控制。

E-mail: jinxin.liu@xjtu.edu.cn

严如强(通信作者), 男, 1975 年出生, 博士, 教授, 博士研究生导师。 主要研究方向为机械故障诊断与智能运维、重大装备振动主控制。

E-mail: yanruqiang@xjtu.edu.cn 